\title{
A Proposed Learner's Data Model: Integrating Informal Learning and Enhancing Personalization and Interoperability
}

\author{
https://doi.org/10.3991/ijet.v16i08.19833 \\ Nissrin Nehiri ${ }^{(凶)}$, Noura Aknin \\ Abdelmalek Essaadi University, Tetouan, Morocco \\ nissrin.nehiri@etu.uae.ac.ma
}

\begin{abstract}
A learner profile is key to personalize learning content. Nowadays learners use different applications and tools to learn (Formal and informal types). Indeed, the diversity of profiles, their content, their structure, their operation, and the actors concerned, limits possible interoperability. Hence, the need for a rich and an interoperable learner profile that describes all previous learning achievements or experiences. In this work, after a brief analysis of available standards in this area, an approach is proposed to build an interoperable learner model based on xAPI statements that combine the formal and informal experiences to enhance learning analytic and personalization. Then, we present a tool to transform collected data into our XML model proposed based on the IMS-LIP standard, and in the end, we explore his utility.
\end{abstract}

Keywords - Informal Learning, Interoperability, Online learning, IMS-LIP, xAPI

\section{$1 \quad$ Introduction}

The interoperability of learners' data is an important issue in the field of IT environments for human learning. Today's learners use different tools to gain experience and knowledge outside the institutional card. For example, since the late 1970s, game-based learning has been an important area of research as stated in Ref [1] and is currently still used by several institutes due to its enormous potential for learning children, adolescents, and even adults. Also, mobile applications have taken a large place in the learning process; several experiences are using mobile technology "as stated in [2]".

Nowadays we find many learning apps, like Blogs, YouTube content, Google Docs, and social interactions on Facebook or Twitter used by students while they are learning or doing homework, living outside the LMS. Teachers are eager to innovate using applications and technologies not supported by the LMS of their establishment and, in so doing; they take their learners out of the institutional context. So, students have to go to several different sites (using different usernames and passwords) in a muddled learning environment. This can create confusion and frustration in the learners. 
On the other hand, these experiences can be used to personalize teaching systems, by planning personalized pedagogical learning paths, thus being useful when looking for an internship or a job for future laureates. This is why, monitoring learners, is a very important phase to meet these needs, as well as interoperability of the learner profile between systems is necessary to integrate the new generation of applications.

In this article, we propose an approach that aims at first, to provide a learner model containing a set of educational information on the experiences of a learner evolving in an online learning environment and other helpful information of the tools from the LMS. This data is collected by the XAPI standard which has been massively implemented by publishers of e-learning solutions in order to ensure interoperability between learning systems [3-4]. Secondly, the design of a tool allowing the transformation of xAPI JSON statements into XML in order to build an interoperable learning model and ensuring the reuse of learners' data.

In the following section, we provide an overview of the research context of our work. Then we present in a general the proposed approach, which allows us to introduce our contributions of this article. Section 4 presents the proposed transformation tools to convert XAPI JSON statement to XML IMS-LIP model. In section 5, we present our IMS-LIP-I as experiment and show in result of our approach. Finally, we conclude the article by highlighting some perspectives that we consider relevant for the rest of this work.

\section{Context of the Research}

This section explains what a learner profile is, what are the main interoperability standards of the learner profile, the impact of informal learning from a methodological and pedagogical point of view, and the usefulness of the XAPI standard in monitoring learners.

\subsection{Learner model and learner modelling standards}

A learner model is a structure that stores information about the learner, expressed in terms of knowledge, skills, learner activities such as purpose, transcription, skills, qualifications, certifications, etc. To facilitate the acquisition of knowledge by a learner, the authors try to adapt the learning path according to each learner. This personalization can be obtained by developing and using a learner model "as present in [5]".

In our work, we wanted to model the user to ensure the interoperability of his data between systems and enrich his profile with other data relating to experiences outside the LMS in order to maintain better personalization.

The main standards that hold information useful for personalization and allow interoperability of learner models are:

PAPI-Learner [6]: Public and Private Information Learner is a IEEE specification that provide an organization of learner data as a first standard. The main objective is to clarify the semantics and syntax of a learning model, which characterizes a learner and his knowledge. It includes six elements described in the table below: 
Table 1. PAPI specification

\begin{tabular}{|l|l|}
\hline \multicolumn{1}{|c|}{ Elements } & \multicolumn{1}{c|}{ Description } \\
\hline Personal & Contains name, address, CNSS number, CIN, all information used by administration. \\
\hline Preferences & Learner's preferences like learning style, physical limitation. \\
\hline Relations & Relationship to other user like classmates, teammates \\
\hline Security & Learner's security (private keys, public keys.) \\
\hline Performance & Learner's history, current works or future (grades.) \\
\hline Portfolio & Accomplishments, works, abilities, achievements. \\
\hline
\end{tabular}

IMS-LIP [7]: IMS Learner Information Package is a model that describes data in 11 categories (see Table 2), based on an XML structure allowing the exchange of data between several learning systems. It ensures all information about objectives, learning path, activities and achievements. There are several works to extend this model compared to the PAPI model, but it does not meet all of the systems' needs in terms of personalization and adaptation.

There are several works to extend this model compared to the PAPI model, but it does not meet all of the systems' needs in terms of personalization and adaptation.

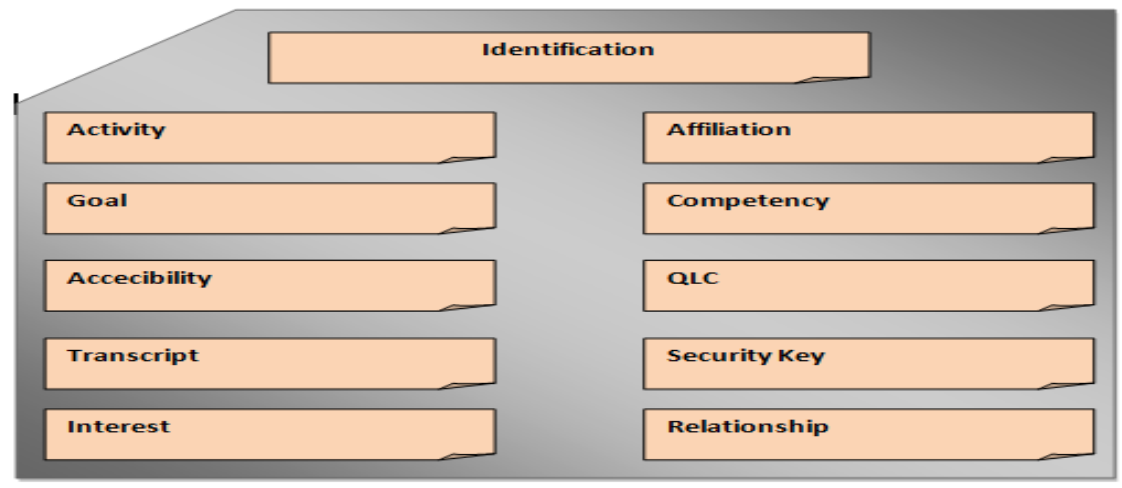

Fig. 1. IMS-LIP standard

\subsection{Informal learning}

Informal learning is understood as the acquisition of knowledge, linked to experience, far from an institution in the course of daily life. It includes a double logic of experimentation and construction of professional identity. As stated in Ref. [8]. distinguishes three types of informal learning according to the intentionality and consciousness of the learner: socialization (tacit learning, "almost natural learning of values, attitudes, behaviours, skills, and knowledge which is produced in everyday life"), fortuitous learning (learning not intentionally sought, co-produced by another activity) and self-directed learning (educational project undertaken without the help of a teacher).

While informal learning is learning that rests primarily in the hands of the learner and involves observation, trial and error, asking for help, conversing with others, listening to stories, reflecting on day events, or being energized by general interests [9]. 
The work presented in Ref. [10]. reported that in the workplace informal learning through questioning, observing colleagues and other uncoordinated and independent learning activities accounts for $80 \%$ of a person's knowledge of this work. However, Cross considers formal and informal learning "to be on a learning continuum" rather than one or the other - or dichotomies.

Today, most of the learning is informal, and if an organization does not tackle informal learning, it leaves a huge amount of learning to chance outside of class boundaries as sited in [8], we use Google, communicate with peers, join online communities, and work together on issues, share learning resources, views, opinions, and ideas. With web 2.0 , social software is widely used for social/collaborative learning. Some of the examples as stated in [9] include wikis, blogs, RSS feeds, podcasts, media sharing, and social tagging. Figure 2 shows the new learning concept.

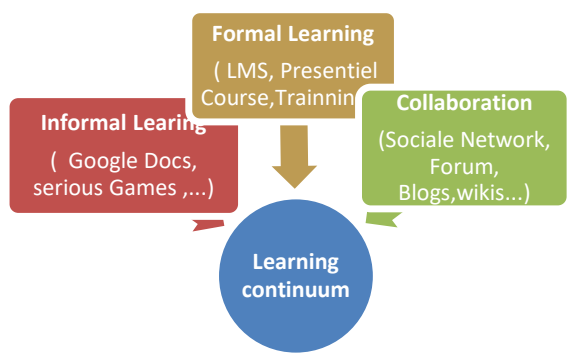

Fig. 2. Learning Continuum

Implementation of this learning continuum in the appropriate way is the current goal of learning practitioners. Indeed, the most appropriate learning solution with this evolution of learning methods would include a mixture of learning in formal and informal contexts. Since there are two situations: training based on basic skills or understanding of concepts which is provided by formal learning programs and a situation when learning needs are of different levels of complexity or when the knowledge base or the content repository is constantly evolving, a more flexible real-time learning culture is needed (Informal).

\section{3 $\quad$ API specification}

In the Experience API is a service that allows for statements of experience to be delivered to and stored securely in a Learning Record Store (LRS). These statements of experience are typically learning experiences, but the API can address statements of any kind of experience. The Experience API is dependent on Activity Providers to create and track these learning experiences [11]. xAPI provides a platform-neutral formalism to collect events occurring in any learning experience. XAPI was released in 2013 as the outcome of an ADL project that aimed to both: (i) improve interoperability between e-learning systems that collect and exchange student learning data, and (ii) overcome the limitations of SCORM. The XAPI specification describes the format to represent discrete learning activities (as JSON statements) and the requirements for Learning 
Record Stores (LRS) that can collect and exchange learner records. The xAPI statement data format is based on WC3 Activity Streams 1.0 with notable changes made to include results and context for a learning activity [12]. The Experience API consists of four interfaces, which are respectively:

- Statement API is responsible for the storage and removal of a statement in LRS

- State API saves the activities in use to buffer cache

- Activity Profile API can refer to the complete description of activities stored in LRS

- Agent Profile API adds data related to the agent to the LRS

- Statement API is responsible for the storage and removal of a statement in LRS

- State API saves the activities in use to buffer cache

- Activity Profile API can refer to the complete description of activities stored in LRS

- Agent Profile API adds data related to the agent to the LRS

\subsection{Synthesis}

Several research projects have been proposed and carried out in the context of interoperability and the collection of learner data. As indicated in Ref [13], the researchers used XAPI to collect the experiences of learners in Remote LAB to improve the analysis of learning. In the field of Architectural Design Teaching [14], the authors exploit the functionalities of this specification to recover the traces of the learners in an interleaving with flash content. A conceptual model has been proposed to assess user behavior via traces traced by xAPI[15].

Subsequently to a review of several researches, we deduce an interoperability scenario to be resolved, namely:

- Integration of informal learning tools in the LMS to improve the personalization of learning content

- Development of an interoperable learner profile, containing formal and informal experiences, with the aim of creating an educational path adapted to the needs and orientations of the learners in order to improve the learning situation, especially in the case that a learner wants to continue studying at another institution.

\section{Proposed Approach for Interoperability Approach}

After describing the technological and methodological context of the problem, we deduce an interoperability scenario to be solved. It aims to integrate the informal experiences of the learner into his institutional profile and ensuring sound. 


\subsection{Overview of the approach}

Securing and reassuring the integration of external tools in LMS is an operation that requires interfaces. In research work, there are several interfaces to do this. For example, Moodle's MNET [16] is a proprietary interface but it limits the tools that can be integrated. In this work and according to our architecture (see Fig.3.) as stated in Ref [17], we propose to use The LTI standard (Learning Tools Interoperability) to ensure the connection of two systems without having to re-authenticate with the destination system.

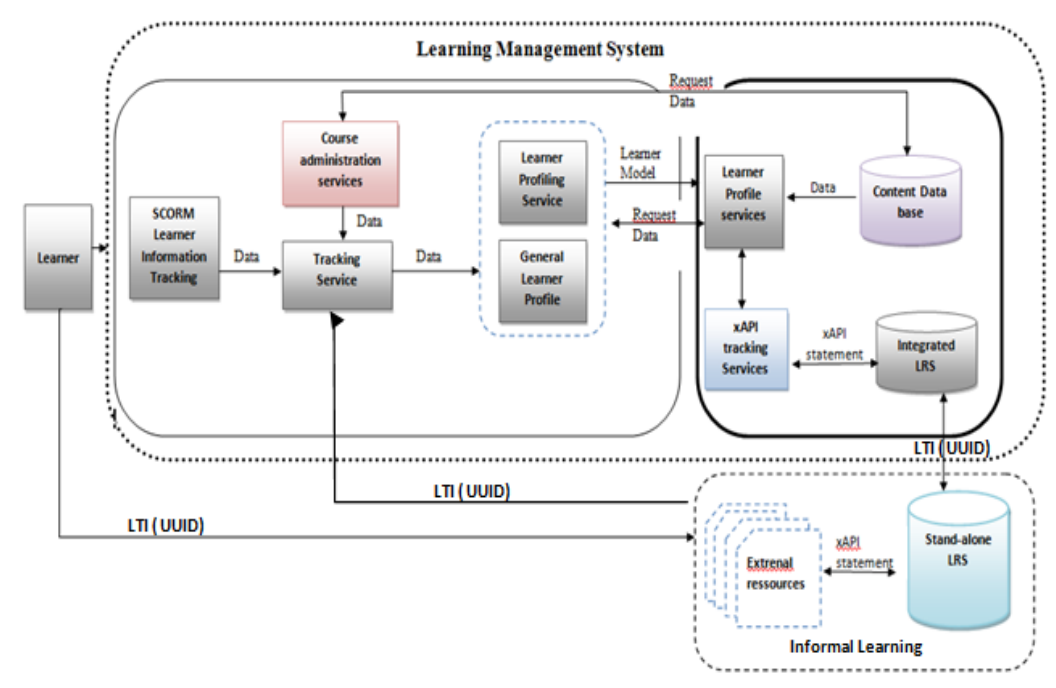

Fig. 3. Proposed architecture of Learning

The approach that we propose consists of three phases which are the collection of the data, the processing, and the elaboration of the final profile. The first phase is data collection, that involves capturing data on learners' experiences, and will then send it as learning records through an XAPI interface to the central LRS. Subsequently, all of the stored data will be exported and translated into XML format via the tool that we have implemented and finally the generation of the learning profile according to the proposed IMS-LIP-I XML scheme. In the following sections, we will see all these phases in detail.

\subsection{Proposed learner model IMS-LIP-I}

The proposed model is the result of analysis of the models presented above (PAPI, IMS LIP), to see if the elements described in the different categories proposed in these models made it possible to exchange our data, and namely the data corresponds to the experiences in the informal sector and outside LMS. However, its elements are not complete enough to cover all the learning data that can be exchanged between e- 
learning systems. We note however in the IMS LIP model a first attempt to model the educational data of the learner. In fact, as stated in Ref. [18] we fin learner's pedagogical pathway added to the category «Activity" of IMS-LIP model.

Our analysis of data at the level of the overall Open and Distance Learning process and new learning concepts allowed us to identify other learning data that can be exchanged between different learning systems, and that can improve the elements of existing learning models, namely activities outside the LMS and those in an informal context. Besides, the formal and informal learning should be connected to optimize learning and that learning is more effective when the learner engages in formal and informal learning activities "as stated in [19]". Furthermore, the results of the study carried out in Ref [20] showed that students perceived the use of blogs as a means of thinking about class topics beyond the weekly class meetings, both individually and in collaboration with peers through blog comments.

As stated, in. Ref. [21]. The IMS-LIP standard is the one chosen to ensure interoperability. Also, it defines a user data model as a set of 11 categories to import or export between systems (see Fig.1.). For those reasons we have chosen it to extend to provide relevant information on activities carried out with external LMS tools and those in the informal context.

The table below illustrates the instances of the new informal activity category added to IMS-LIP.

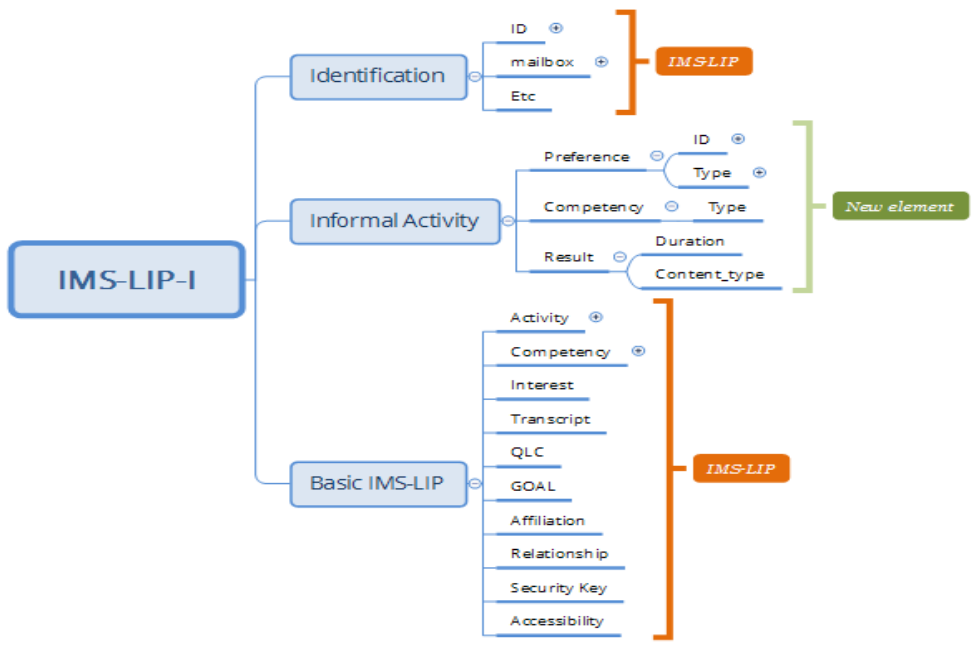

Fig. 4. Proposed IMS-LIP-Informal

The table below shows the description of the added elements: 
Table 2. Informal activity new category of IMS-LIP

\begin{tabular}{|l|l|l|}
\hline New category & Main elements & \multicolumn{1}{c|}{ Description } \\
\hline Informal Activity & Preference & $\begin{array}{l}\text { This element describes all the technologies and tools used by the } \\
\text { learner during informal learning (Social media, Blogs.) }\end{array}$ \\
\cline { 2 - 3 } & Competency & $\begin{array}{l}\text { This component contains the skills to be acquired with the use of non- } \\
\text { LMS resources and tools for informal learning. }\end{array}$ \\
\cline { 2 - 3 } & Result & $\begin{array}{l}\text { This element contains the results, which are the reports, the time spent } \\
\text { on its tools. These results give the first analysis of learners' behaviors } \\
\text { which helps to personalize the educational path. }\end{array}$ \\
\hline
\end{tabular}

\subsection{Collection of learner data}

The operations of the collection and recording system are summarized in the figure below that represents the data flow existing between LMS, LRS, and external tools (Informal). The green flow represents the LMS experience management data: the educational information of experience. The blue flow represents endogenous data in an external context of the LMS, such as the activities carried out in Google Docs, serious games.

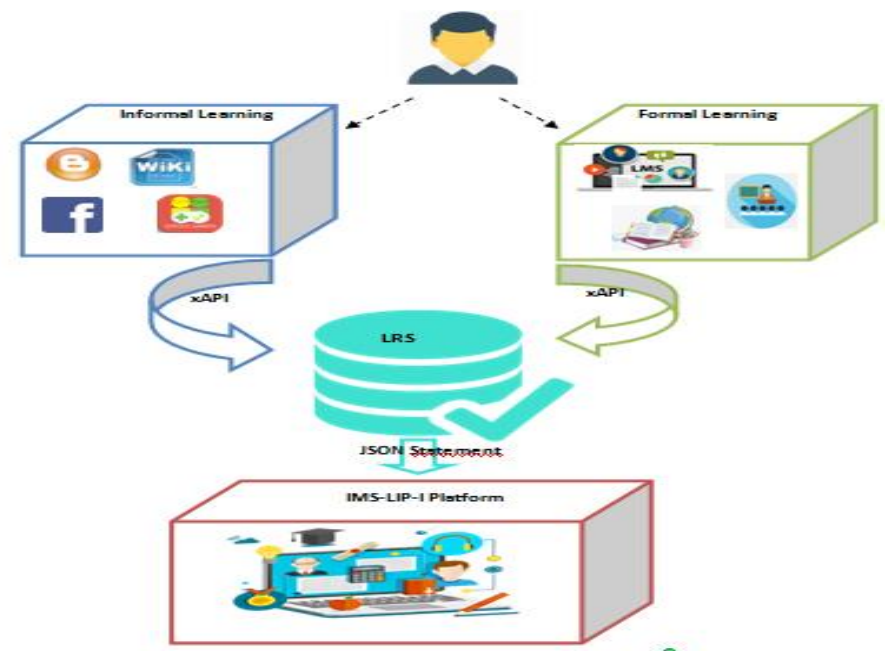

Fig. 5. Collection learner experiences

\section{The systems:}

- Formal Learning (LMS): A system that delivers content to users and manages access.

- LRS: System which stores in the form of statements all the information produced by the interaction of users with the content. It interacts with the LMS and can be inside or outside the LMS.

- Informal learning tools: External tools allowing the learner to acquire experiences outside LMS. 


\section{The roles:}

- Learner: An individual who interacts with content.

- Administrator: Individual or organization that makes content available to an learners. Subsequently, it aggregates and interprets a set of data resulting from content exploitation.

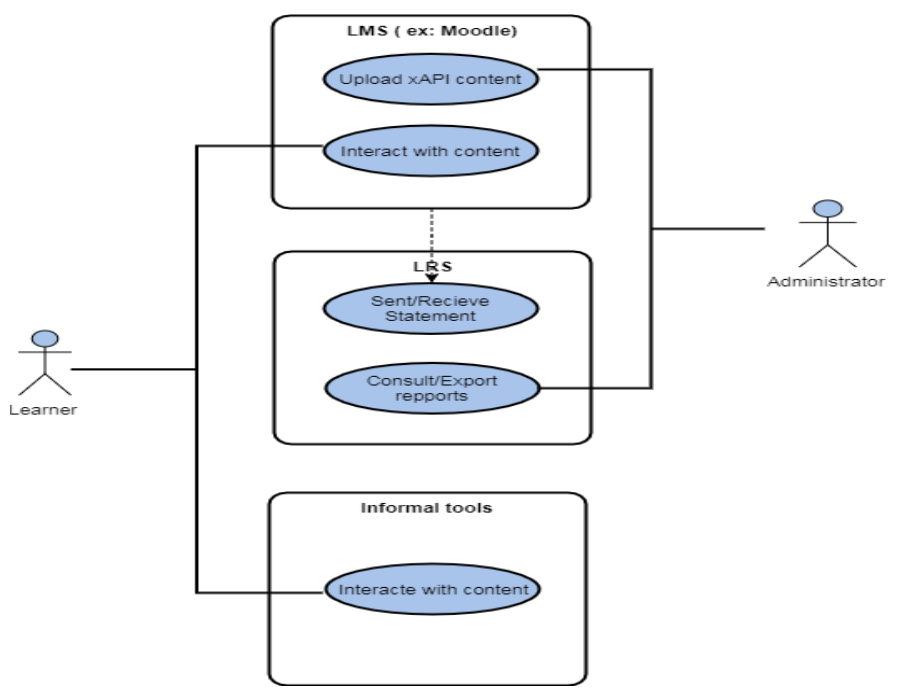

Fig. 6. Use case diagram

\section{Uses:}

- Upload of xAPI compliant content: Get an authoring tool to produce a package that complies with the XAPI standard, that is to say, that sends significant statements to an LRS according to the established protocol.

- Interact with LMS content: For example, navigate through a hypertext, take a test, and explore interactivity. The content can also retrieve statements in order to ensure persistence between a user's work sessions or to allow a sharing between users.

- Produce and consult reports: This is the GET section of the "Statement API", which allows you to produce reports either in the LRS or via a specialized application.

- Interact with Informal content: Interactions with informal learning tools such as social networks, wikis, etc.

\section{Trace models:}

The traces emitted to the LRS are statements xAPI, the fact transmission via a Web service REST (Representational State Transfer) based on the JavaScript object notation (JSON) for its data format. The basic structure of an xAPI trace is in the following format: 
$\langle$ actor $\rangle\langle$ verb $\rangle<$ context $><$ object $><r e s u l t>$

The main variables used to monitor these actions are already defined by the Tin Can standard (xAPI). In general, the basic variables to represent an action are the time when the action took place, the user who was the interpreter, the action which was executed, the object of the action (if applicable), and the results produced (if applicable).

\section{Processing Data with the Proposed JSON to XML Tool}

The traces collected will be processing by the transformation tools. In object to use this tool as an independent application, we had design it as a module. It has two modules: the first module transforms the JSON XAPI statement into XML. The second ensures the structuring of the traces according to the XML scheme of the IMS-LIP-I proposed and explore them in a platform to give a report to the tutor, learner and to choose which information to be exchanged.

\subsection{Transformation module}

The data collected is stored in the LRS are in JSON format, after the export of these traces, a transformation is required to integrate the missing elements of our proposed model, namely those of the informal activity. Indeed, the aim is to enrich the learning profile with information absent from the informal domain. This proposal was motivated by the need to remedy some interoperability gaps. Certainly, the XML language provides a structured document, offers powerful functionalities, namely import and export between different systems, as well as the readability and reusability of data.

The administrator uploads the JSON file to convert. Then, he has the possibility to export all data into XML format.

Example of the transformation of an activity captured by XAPI:

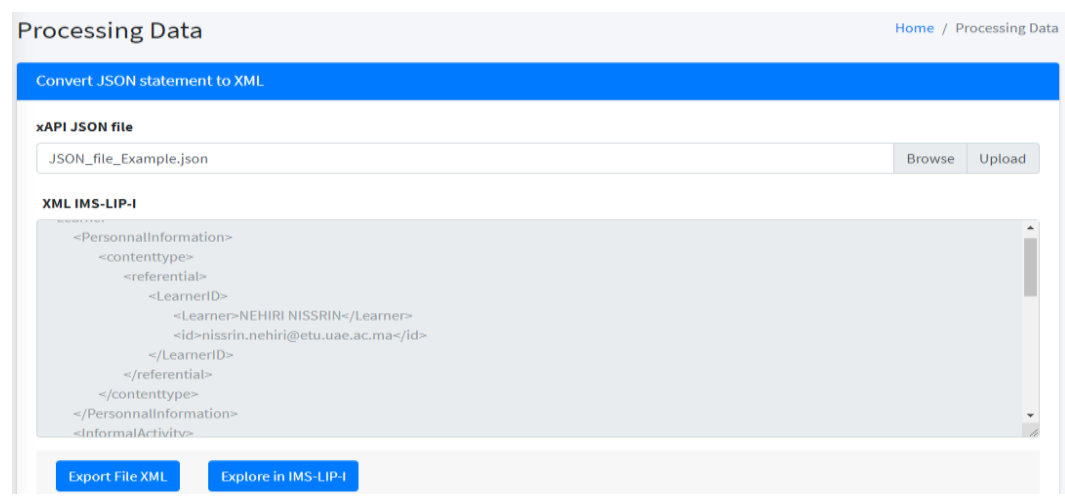

Fig. 7. XAPI Json to XML IMS-LIP 


\subsection{IMS-LIP-I generator module}

In our solution, a learner model is composed of a reference model and one or more temporary context components. This way of doing things makes it easier for us to manage the evolution of learner experiences over time.

The reference model is the IMS-LIP-I model proposed. It inherits the same categories from IMS-LIP with a new category that describes the learner's experiences outside of LMS. This model is linked to one or more components of the learner's temporary context which describe the learning and experiences linked to a particular training or context.

The temporary context components are formal context components linked to a particular training (such as a course, etc.) and informal context components linked to experience in an environment other than LMS (Google docs, Remote Lab, etc.). With each training course followed by a learner, a temporary context component is created via all the xAPI statements stored in an LRS.

The figure below illustrates the conceptual model of the IMS-LIP-I model.

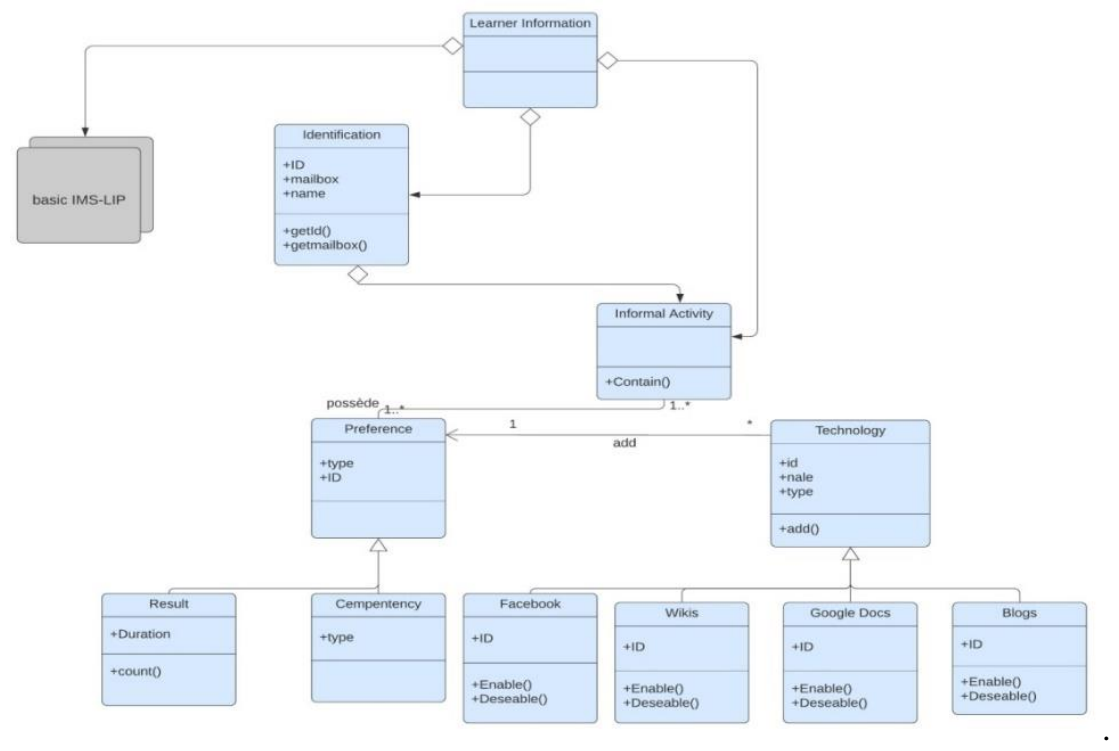

Fig. 8. Class diagram of proposed model

\section{$5 \quad$ Experimentations and Result}

\subsection{IMS-LIP-I platform}

We have designed and produced an IMS-LIP-I platform using the PHP language to evaluate and validate our approach. This application allows a collection of learner models in XML format and offers the possibility for the teacher, student to explore the data 
of the registered learner in the categories of the model that we have proposed as well as functionality to choose the data to be exchanged with other learning platforms. The teacher/learner makes his choice between the different components of LIP-I. These elements appear as list of choice, then he exports the data in XML files, that allows the re-use and interoperability of information.

This platform gives to the tutor a report of all experiences devoted by the learner, so he can show some indicators for personalization and adaptation for the future learning objects.
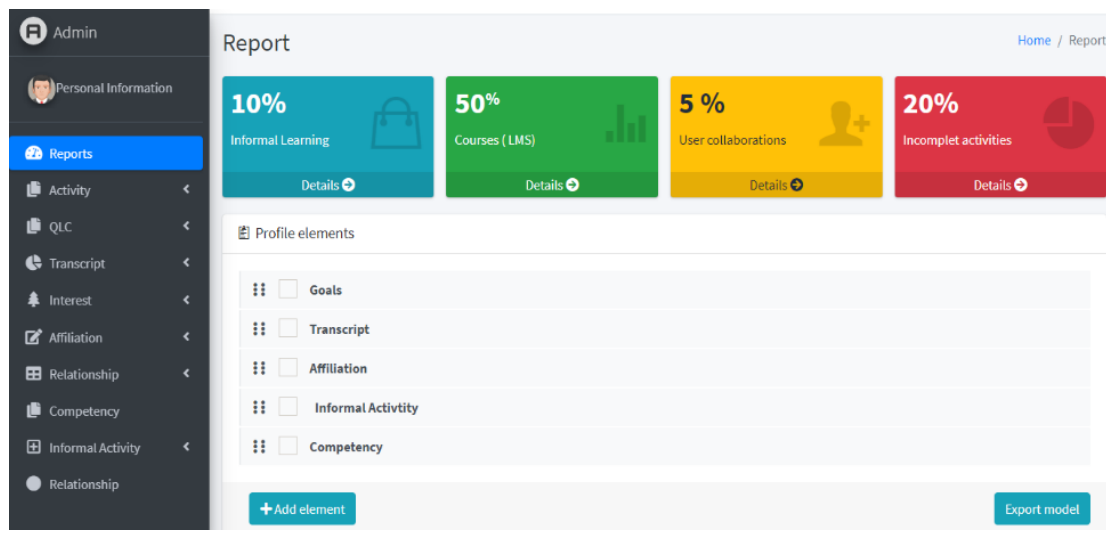

Fig. 9. IMS-LIP-Informal platform

After the transformation of the JSON data into XML, scripting of the data is made according to the XML structure. The elements of IMS-LIP-I are optional.

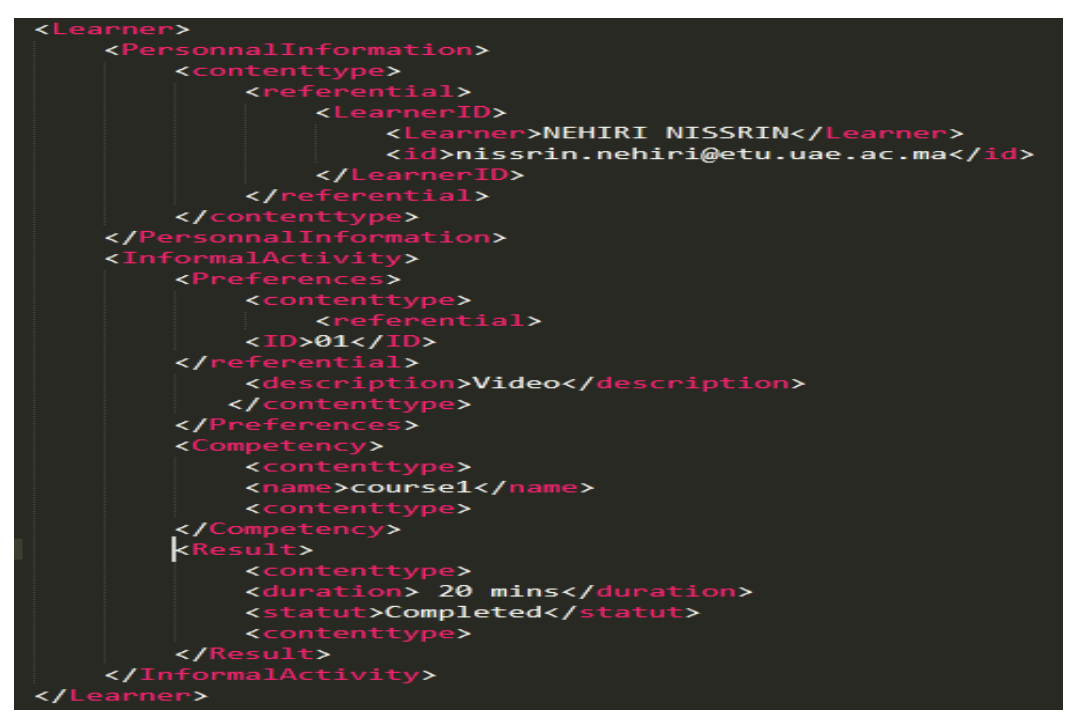

Fig. 10. IMS-LIP-Informal XML model 


\subsection{Utility}

In the area of learning, there is a need to take training or complete training in another learning platform. So, to allow the learner to continue his learning activities, a rich and interoperable profile facilitates this need.

The approach that we have proposed presents an architecture for the development of a learning model rich in XML format, which describes and groups all the information concerning the learner, namely that of the formal and informal context on one side, and in the other. 'on the other hand, a model in XML format facilitating interoperability and data exchange with other platforms and learning systems. The use of this type of learning model ensures a common basis of exchange in similar situations. Its implementation requires a plugin which makes it possible to read the data recorded in the XML schema and dispatch them in the appropriate place at the level of the database of the learning system (LMS).

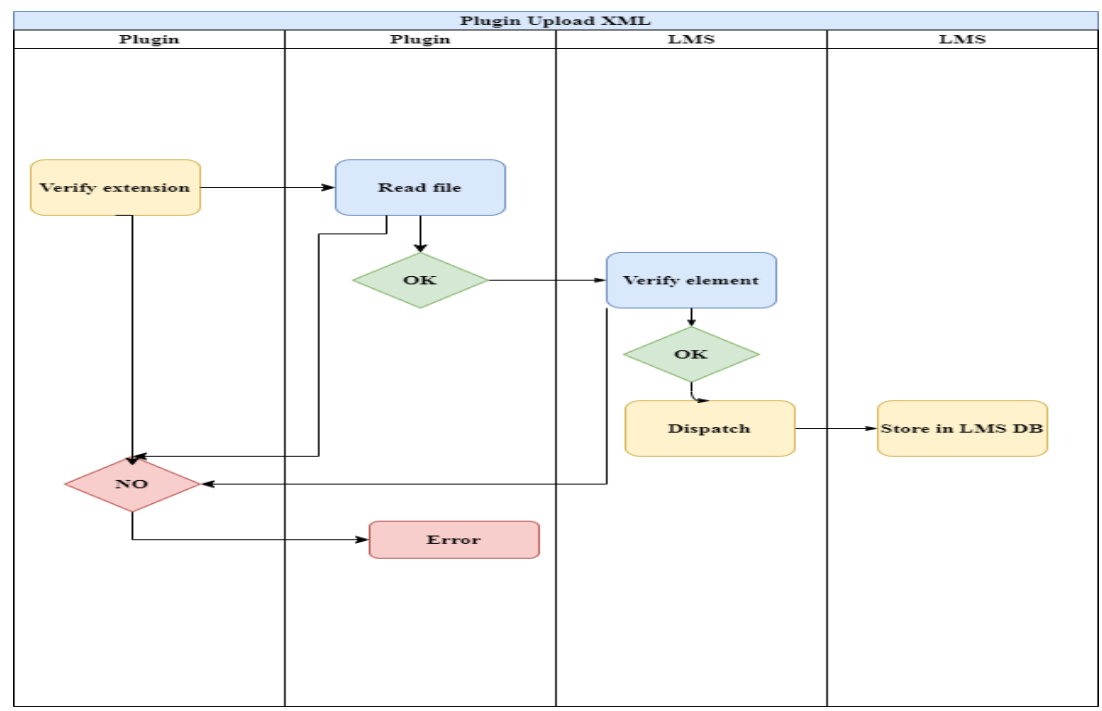

Fig. 11. Process diagram of XML reader plugin

\section{Conclusion}

Share and reuse learner data between different learning system is currently available with the learning technologies, but with this grow up of learning teaching and tools, learners acquire many experiences, so it is necessary to find and collect those information and integrated them in the learner profile in order to personalize learning path and enhance learning analytic.

In this sense, our proposal shows how to ensure seamless integration of formal and informal learning tools that aims to enrich learner profile and ensure interoperability between systems. As future work, we plan to consider the deployment of learning 
analytic tools in order to get a feedback of the use of informal learning tools in the context of LMS.

\section{$7 \quad$ References}

[1] Aguilera de, M. and Mendiz, A. (2003) 'Video games and education: (education in the face of a 'parallel school')', Computers in Entertainment (CIE), Vol. 1, pp.1-10. https://doi. org/10.1145/950566.950583

[2] Brown-Martin, G. (2008) 'It's the learning, stupid!', in Arnedillo, I. and Isaias, P. (Ed.): IADIS International Conference Mobile Learning, IADIS Press, pp.17-19.

[3] L.F. Zapata Rivera, M. M. Larrondo Petrie, Models of Collaborative Remote Laboratories and Integration with Learning Environments, IJEO journal. http://dx.doi.org/10.3991/ ijoe.v12i09.6129, vol 12, No 09 (2016). https://doi.org/10.3991/ijoe.v12i09.6129

[4] Haiou Wang, Construction of xAPI-based Multimedia Interaction Technology in Architectural Design Teaching,IJEO journal, Vol 12, No 07 (2017)

[5] D. Py, " Some artificial intelligence methods for student modeling ", Educational sciences and techniques, Vol. Vol. 5, $\mathrm{n}^{\circ}$ 2, 1998.

[6] IEEE LTSC: Ltsc public and private information for learners, http://www.cenltso.net/main.aspx?put $=230$

[7] IMS GLC: IMS Learner Information Packaging Information Model Specication

[8] Daniel Schugurensky, «Vingt mille lieues sous les mers »: les quatre défis de l'apprentissage informel », Revue française de pédagogie, 160 | 2007, p 13-27. https://doi.org/10.4000/ rfp. 583

[9] Cross, J. (2007). Informal learning: Rediscovering the natural pathways that inspire innovation and performance. San Francisco, CA: Pfeiffer reference

[10] Attwell, Graham (2007). The Personal Learning Environments - the future of eLearning? eLearning Papers, vol. 2 no. 1. ISSN 1887-1542.

[11] Experience API. Avialable: GitHub - adlnet/xAPI-Spec [Accessed: Apr. 12, 2017].

[12] Megan BoweTin Can vs. Activity Streams. Avialable: http://tincanapi.com/tin-can-vs-activity-streams/[Accessed: Apr. 12,2017]

[13] L.F. Zapata Rivera, M. M. Larrondo Petrie, Models of Collaborative Remote Laboratories and Integration with Learning Environments, IJEO, Journal, Vol 12, No 09 (2016), https:// doi.org/10.3991/ijoe.v12i09.6129

[14] Haiou Wang, Construction of xAPI-based Multimedia Interaction Technology in Architectural Design Teaching,IJEO journal , Vol 12, No 07 (2017)

[15] Mustafa Man, Mohd Hafriz Nural Azhan, Conceptual Model for Profiling Student Behavior Experience in e-Learning,IJET, journal, Vol. 14, No. 21, 2019.

[16] A. Büchner, "Moodle 3 Administration", Packt Publishing Ltd, 2016.

[17] N. Nehiri, N. Aknin, "New architecture to exchange learner's data between e-learning systems using xapi," In Proc. International Conference on Technology, Education and Development'11, 2017, pp 9611-9617. https://doi.org/10.21125/inted.2017.2267

[18] Lahcen Oubahssi, Monique Grandbastien. Un modèle pour améliorer l'interopérabilité des données pédagogiques apprenant. 3ième conférence en Environnement Informatique pour l'Apprentissage Humain - EIAH 2007, Jun 2007, Lausane, Suisse. pp.437-448. ffhal00161587.

[19] Hall, R. (2009). Towards a fusion of formal and informal learning environments: The impact of the read/write web. Electronic Journal of e-Learning, 7(1), 29-40. 
[20] Harrison, D. (2011). Can blogging make a difference? https://campustechnology.com/articles/2011/01/12/can-blogging-make-a difference.aspx

[21] Ehlers, U.D., Pawlowski, J.M.: Handbook on quality and standardisation in e- learning [electronic resource]. Springer (2006)

\section{Authors}

Nissrin Nehiri received the Master degree in Computer Science in 2014 from Abdelmalek Essaadi University in Tetuan, Morocco. Currently, he is a PhD Candidate and member of Information Technology and Modelling Systems Research Unit at the same university.

Noura Aknin Professor of Electrical \& Computer Engineering at Abdelmalek Essaadi University since 2000. She received $\mathrm{PhD}$ degree in Electrical Engineering in 1998. She is the Head of Research Unit Information Technology and Modelling Systems. She is the Co-founder of the IEEE Morocco Section since November 2004 and a member of several IEEE societies. Noura AKNIN is R\&D project manager/member related to new technologies and their applications. She was a chair of several conferences and she has been involved in the organizing and in the Scientific Committees of several international conferences held worldwide dealing with computer science and applications. (noura.aknin@uae.ac.ma)

Article submitted 2020-11-14. Resubmitted 2020-12-15. Final acceptance 2020-12-16. Final version published as submitted by the authors. 